\title{
What we have here is a failure to fertilize: back to basics
}

\author{
David F. Albertini ${ }^{1}$
}

Published online: 17 June 2015

(C) Springer Science+Business Media New York 2015

Familiar to fans of the late actor Paul Newman, the 1967 cult classic film "Cool Hand Luke" is known for the following reprisal uttered by the road prison captain:

"What we got here is a failure to communicate."

Despite all efforts taken in the ART laboratory to make the dialogue between egg and sperm fulfilling as a means to embryo production, failure to fertilize continues to be an obstacle thwarting the hopes of many patients in their quest of parenthood. Even the introduction of ICSI some 20 years ago, while measurably improving the efficiency of egg activation and embryo production, remains inadequate in a minority of cases at effecting the signaling pathways that we now appreciate are the hallmarks of human fertilization. ICSI itself has evolved over the years as a means for dealing with many of the most challenging cases of male infertility-from tree-trimming the flagellum to taking the role of PLC $\zeta$ out of the "hands" of the already deconstructed spermatozoon by spiking injection medium with calcium - all modifications of the original procedures that derived from a solid foundation in reductionistic basic science. But even the injection pipette with its cell

Capsule Despite a deepening understanding of the molecular underpinnings of fertilization in humans, the processes that launch the earliest steps in the pathway to embryogenesis continue to elude management and explanation for the many patients awaiting good news the day after egg retrieval. This issue of JARG brings to surface some of the more fundamental aspects of egg activation empowered by the motility properties of sperm that offer clinically tractable solutions to the problem of fertilization failure.

David F. Albertini

dalbertini@kumc.edu

1 University of Kansas Medical Center, Kansas, KS, USA (castrated as it is) and ionic contents remain incompetent to fertilize some eggs. Why?

Our issue of JARG this month features conundrums of fertilization with immediate clinical implications. And, in what we are sometimes able to remind our readership of, the mechanisms underscoring male infertility and fertilization failure have overlooked a historical framework that is often clouded in importance and worthy of reinterpretation in light of recent findings.

Begin with our cover this month. Tracing over a century's worth of discovery, this montage displays both the familiar (outer edges) and hidden (center) underpinnings of the sperm tail or flagellum with which the male gamete obtains its ticket to ride towards destiny. Among the many microtubules that comprise the axonemal cytoskeleton is a subclass of biochemically unique filaments made of subunits of proteins known as tektins - the fine filaments shown on the cover. Tektins became suspect in being prime movers of human sperm in a paper published in JARG just over 2 years ago with increased levels of this protein associated with improved fertilization rates and embryo quality (Bhilawadikar et al., JARG 2013, 30:513-523, DOI 10.1007/s10815-013-9972-6). For more on tektins and axonemal motility, we ask our readers to look out for detailed coverage of this topic in an upcoming issue. For the "failed fertilization" fans among you, you need not look any further than what lies ahead.

From sperm tail proteomics, to a mutational analysis of sperm motility, to matters well in excess of the predictable and oft implicated PLC zeta sparking during egg activation, a collection of thought-provoking papers that touch upon various aspects of the enigma of human fertilization lead of this issue. For more suspicion and intrigue, take a close look at the contributions by Vassena and colleagues whose results highlight that even in cases of proven male fertility, molecular players other than those typically involved in egg activation 
may have escaped detection and will prompt more questions as to the origins of failed fertilization (PLC $\zeta$ disruption with complete fertilization failure in normozoospermia 10.1007/ s10815-015-0496-0).

And does it matter where sperm are placed during ICSI? That the sperm entry point determines patterns of calcium release that elicit the cortical reaction has been recognized for many years. This has led others to suggest that the organelles responsible for generating and responding to bursts of intracellular calcium release are topographically heterogeneous - that is, subcortical or deep within the ooplasm. The results of De Vos and colleagues demonstrate that sperm positioning during ICSI is an unlikely suspect in cases of failed fertilization (Deposition of the spermatozoon in the human oocyte at ICSI: impact on oocyte survival, fertilization and blastocyst formation 10.1007/s10815-015-0482-6).

An interesting case report from Ebner and colleagues adds yet another twist to the puzzle (Healthy twin live-birth after ionophore treatment in a case of theophylline-resistant
Kartagener syndrome 10.1007/s10815-015-0486-2). Depending on your perspective, cases like this bring out the best and the worst of human ARTs.

Returning to the classics will remind those old enough to recall that it was the cell biological investigation of patients with Kartagener's syndrome by Afzelius that first illuminated the role of dynein arms in human cell biology, given the flagellar and ciliary dyskinesia that underlies their disease conditions. And, not surprisingly, it was the field of human ARTs that demonstrated the "correctability" of complete male infertility in these patients using ICSI, rectifying infertility in such patients but assuring that some if not all of the deleterious syndromic consequences be inherited. What Ebner and colleagues reveal here, however, is about more than clinical success. Rather, it is about defining modifications in treatment strategies that concurrently advance our knowledge of human fertilization and open new avenues for research into the subcellular complexity of the earliest stages of development. 